University of South Carolina

Scholar Commons

$10-1-2012$

\title{
Atomistic Investigation of Scratching-Induced Deformation Twinning in Nanocrystalline $\mathrm{Cu}$
}

Junjie Zhang

Tao Sun

Yoganda Yan

Dong Shen

Xiaodong Li

University of South Carolina - Columbia, lixiao@cec.sc.edu

Follow this and additional works at: https://scholarcommons.sc.edu/emec_facpub

Part of the Applied Mechanics Commons, and the Other Mechanical Engineering Commons

\section{Publication Info}

Published in Journal of Applied Physics, Volume 112, Issue 7, 2012, pages \#073526-.

(C) Journal of Applied Physics 2012, American Institute of Physics.

Zhang, J., Sun, T., Yan, Y., Shen, D., \& Li, X. (1 October 2012). Atomistic Investigation of Scratching-Induced Deformation Twinning in Nanocrystalline Cu. Journal of Applied Physics, 112 (7), http://dx.doi.org/ $10.1063 / 1.4757937$

This Article is brought to you by the Mechanical Engineering, Department of at Scholar Commons. It has been accepted for inclusion in Faculty Publications by an authorized administrator of Scholar Commons. For more information, please contact digres@mailbox.sc.edu. 


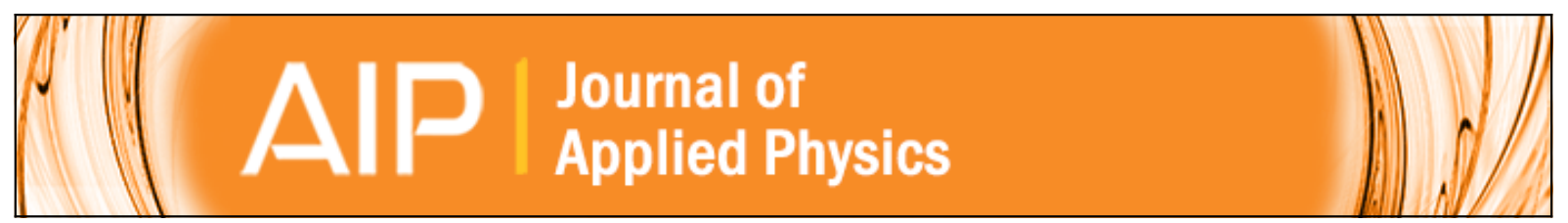

Atomistic investigation of scratching-induced deformation twinning in nanocrystalline Cu

Junjie Zhang, Tao Sun, Yongda Yan, Dong Shen, and Xiaodong Li

Citation: Journal of Applied Physics 112, 073526 (2012); doi: 10.1063/1.4757937

View online: http://dx.doi.org/10.1063/1.4757937

View Table of Contents: http://scitation.aip.org/content/aip/journal/jap/112/7?ver=pdfcov

Published by the AIP Publishing

\section{Articles you may be interested in}

A comparative study on shock compression of nanocrystalline Al and Cu: Shock profiles and microscopic views of plasticity

J. Appl. Phys. 114, 163504 (2013); 10.1063/1.4826624

Size effects of primary/secondary twins on the atomistic deformation mechanisms in hierarchically nanotwinned metals

J. Appl. Phys. 113, 203516 (2013); 10.1063/1.4808096

Nucleation of deformation twins in nanocrystalline face-centered-cubic metals processed by severe plastic deformation

J. Appl. Phys. 98, 034319 (2005); 10.1063/1.2006974

Formation mechanism of fivefold deformation twins in nanocrystalline face-centered-cubic metals Appl. Phys. Lett. 86, 103112 (2005); 10.1063/1.1879111

Deformation twinning in nanocrystalline copper at room temperature and low strain rate Appl. Phys. Lett. 84, 592 (2004); 10.1063/1.1644051

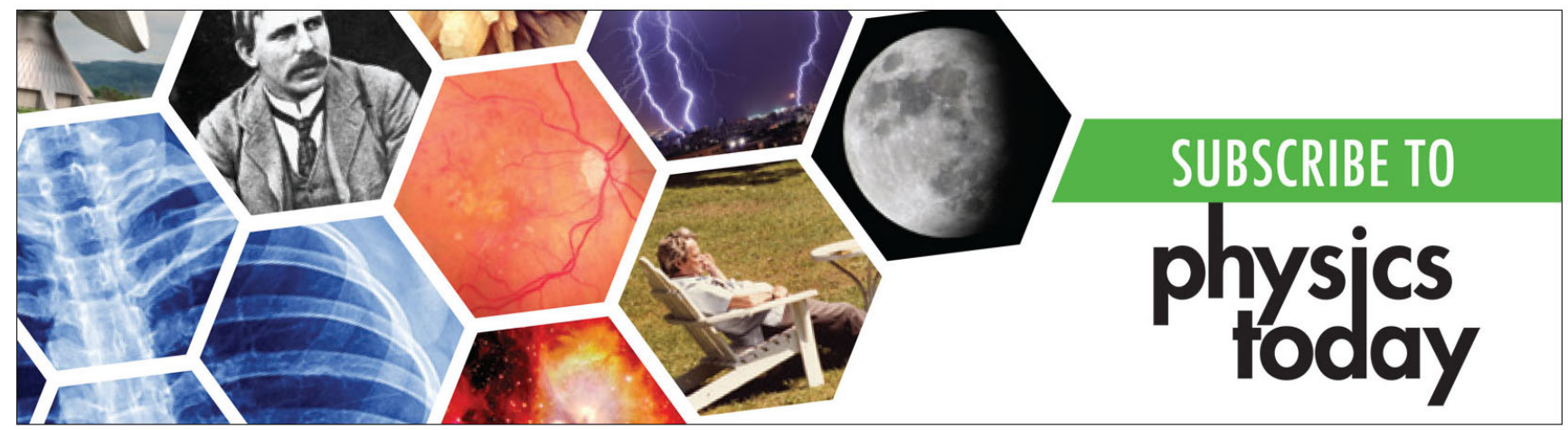




\title{
Atomistic investigation of scratching-induced deformation twinning in nanocrystalline $\mathrm{Cu}$
}

\author{
Junjie Zhang, ${ }^{1,2}$ Tao Sun, ${ }^{1}$ Yongda Yan, ${ }^{1}$ Dong Shen, ${ }^{1}$ and Xiaodong $\mathrm{Li}^{3, \mathrm{a}}$ ) \\ ${ }^{1}$ Center for Precision Engineering, Harbin Institute of Technology, P.O. Box 413, 150001 Harbin, \\ People's Republic of China \\ ${ }^{2}$ Interdisciplinary Centre for Advanced Materials Simulation (ICAMS), Ruhr-University Bochum, \\ Universitätsstraße 90a, 44789 Bochum, Germany \\ ${ }^{3}$ Department of Mechanical Engineering, University of South Carolina, 300 Main Street, Columbia, \\ South Carolina 29208, USA
}

(Received 6 May 2012; accepted 10 September 2012; published online 11 October 2012)

\begin{abstract}
Deformation twinning is an important deformation mode of nanocrystalline metals. In current study, we investigate the scratching-induced deformation twinning in nanocrystalline $\mathrm{Cu}$ by means of molecular dynamics simulations. The tribological behavior, the deformation mechanisms, the formation mechanism of deformation twins, and the grain size dependence of the propensity of deformation twinning are elucidated. Simulation results demonstrate that deformation twinning plays an important role in the plastic deformation of nanocrystalline $\mathrm{Cu}$ under nanoscratching, in addition to dislocation activity and grain boundary-associated mechanism. The nucleation of initial twinning partial dislocations originates from the dissociation of lattice partial dislocations that emit from grain boundary triple junctions, and subsequent twin boundary migration is resulted from the glide of lattice partial dislocations emitted from twin boundary-grain boundary intersections on the twin plane. It is found that the propensity of deformation twinning in nanocrystalline $\mathrm{Cu}$ under scratching has strong dependence on both grain size and stress state. These findings will advance our understanding of the tribological behavior of nanocrystalline $\mathrm{Cu}$ and provide design and fabrication guidelines for nanocrystalline $\mathrm{Cu}$ based micro/nanosystems. (C) 2012 American Institute of Physics. [http://dx.doi.org/10.1063/1.4757937]
\end{abstract}

\section{INTRODUCTION}

Nanocrystalline materials with internal microstructural size down to nanoscale regime have drawn wide range of interests due to their unique mechanical properties. ${ }^{1,2}$ To facilitate the rational design and application of nanocrystalline materials, fundamental investigations of their deformation behavior under external loads are essentially required. In addition to dislocation slip and grain boundary (GB)associated mechanism, deformation twinning has been demonstrated to be an important deformation mechanism operating in the plastic deformation of nanocrystalline facecentered-cubic (FCC) metals subjected to mechanical loading at low temperature and high strain rate. ${ }^{3-12}$ In particular for the nanocrystalline $\mathrm{Cu}$ under tension, torsion, or rolling modes, deformation twinning plays an important role in the plastic deformation because of the low stacking fault energy. ${ }^{13-17}$ Although several twinning mechanisms have been proposed, our understanding of the relation between deformation twinning and the resultant mechanical behavior is far from being completed. On the other hand, little attention has been paid to the role of deformation twinning playing in the tribological behavior of nanocrystalline $\mathrm{Cu}$. It has been proven that the stress state, in addition to other external parameters such as temperature and strain rate, has a remarkable influence on the twinning stress at which a mechanical

\footnotetext{
a) Author to whom correspondence should be addressed. Electronic mail: lixiao@cec.sc.edu.
}

twin nucleates. ${ }^{18}$ During tribological test, the specimen undergoes localized elastic and plastic deformations under multi-axial stress states, which is distinctly different from the uniform deformation under uniaxial stress state in tensile test. Therefore, a key question is raised, but not well answered: can mechanical twins be formed in nanocrystalline $\mathrm{Cu}$ subjected to nanoscratching? While friction and wear are of critical importance for the application of nanocrystalline materials, a fundamental understanding of the relationship between the scratching-induced deformation twinning and the resultant tribological behavior should provide critical guidelines for the design, manufacturing, and operation of nanocrystalline $\mathrm{Cu}$ based micro/nanosystems.

It has been demonstrated that the influence of internal microstructure on the deformation behavior of nanocrystalline metals is size dependent. ${ }^{17,19,20}$ In particular, the grain size dependence of deformation twinning in nanocrystalline $\mathrm{Cu}$ is not monotonous. Previous studies showed that the twinning stress in nanocrystalline FCC metals increases with decreasing grain size, i.e., the grain size-dependent propensity of deformation twinning follows a reverse Hall-Petchtype relationship. ${ }^{21,22}$ However, a recent report claimed that the propensity of deformation twinning exhibits a normal Hall-Petch grain size dependence upon further decreasing grain size of nanocrystalline $\mathrm{Cu}^{23}$ Therefore, it is also intriguing to investigate the effect of grain size on the propensity of scratching-induced deformation twinning.

In this study, we perform molecular dynamics simulations to investigate the scratching-induced deformation 
twinning in nanocrystalline $\mathrm{Cu}$. Atomic configurations of three-dimensional randomly oriented nanocrystalline $\mathrm{Cu}$ samples are utilized. The tribological response, the deformation mechanisms, and the formation mechanism of deformation twins are elucidated. Furthermore, the effect of grain size on the deformation behavior of $\mathrm{Cu}$ samples is also studied. In particular, the physical mechanism for the observed deformation twins is unraveled in terms of the dissociation of lattice partial dislocations, twin boundary (TB) migration, and Hall-Petch grain size dependence of deformation twinning. Based on such atomistic simulations, we demonstrate that deformation twinning is an important deformation mode in nanocrystalline $\mathrm{Cu}$ subjected to nanoscratching.

\section{SIMULATION METHOD}

Figure 1 illustrates the MD model of nanoscratching utilized in this study, which consists of a nanocrystalline $\mathrm{Cu}$ sample of full atomic representation and a virtual spherical probe. Three nanocrystalline $\mathrm{Cu}$ samples with different average grain sizes of 16,13, and $10 \mathrm{~nm}$, namely Sample 1, 2, and 3 for simplicity, respectively, are prepared by using the Voronoi diagram. Each $\mathrm{Cu}$ sample has the same dimension of 21.5, 14.5, and $21.5 \mathrm{~nm}$ in $\mathrm{X}, \mathrm{Y}$, and $\mathrm{Z}$ directions, respectively. Periodic boundary conditions are applied in laterally $\mathrm{X}$ and $\mathrm{Z}$ directions of each $\mathrm{Cu}$ sample, and the bottom of which is fixed to prohibit rigid movement of the sample during nanoscratching process. The atomic interactions in nanocrystalline $\mathrm{Cu}$ samples are described by an embedded atom method (EAM) potential parameterized for $\mathrm{Cu}^{24}$ Prior to nanoscratching, the as-created nanocrystalline $\mathrm{Cu}$ samples are relaxed to their equilibration configurations by following procedures: atoms in samples are first relaxed to their minimum energy configurations using the fast inertia relaxation engine (FIRE) algorithm, ${ }^{25}$ and then the samples are heated up to $30 \mathrm{~K}$ under 0 bar using the Nose-Hoover thermostat for $50 \mathrm{ps}$ in the isothermal-isobaric (constant number of particles $(\mathrm{N})$, constant pressure $(\mathrm{P})$ and temperature $(\mathrm{T})$,

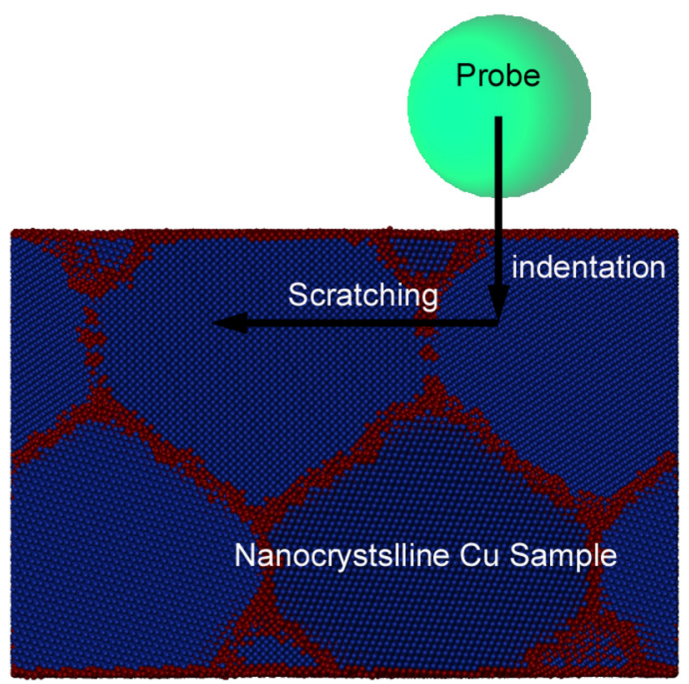

FIG. 1. MD model of nanoscratching on nanocrystalline $\mathrm{Cu}$. The atoms in $\mathrm{Cu}$ sample are colored according to calculated CNA value. The virtual spherical probe is represented by a repulsive potential.
NPT) ensemble. Note that the low temperature of $30 \mathrm{~K}$ is selected to diminish the thermally activated dislocation motion and promote twinning tendency. ${ }^{12,26}$

The well-equilibrated nanocrystalline $\mathrm{Cu}$ samples are then subjected to nanoscratching using a spherical probe with a radius of $4 \mathrm{~nm}$ in the microcanonical (constant number of particles (N), constant volume (V) and energy (E), NVE) ensemble. The probe is modeled by a strong repulsive potential. ${ }^{27}$ Figure 1 depicts that the nanoscratching process consists of two sequential stages, as indentation and following scratching, respectively. In indentation stage, the probe penetrates into each $\mathrm{Cu}$ sample by $2.24 \mathrm{~nm}$ along negative $\mathrm{Y}$ direction at a constant velocity of $20 \mathrm{~m} / \mathrm{s}$, and then it scratches $4.07 \mathrm{~nm}$ along negative $\mathrm{X}$ direction at a constant velocity of $20 \mathrm{~m} / \mathrm{s}$ in the following scratching stage. It should be noted that the simulated nanoscratching velocity is few orders of magnitude higher than experiments, because of the requirement of obtaining large strain in the plastic deformation within reasonable computational time. All the MD simulations are completed using the classical MD package-ITAP Molecular Dynamics (IMD) with a time step of $1 \mathrm{fs} .{ }^{28}$ The lattice defects generated during nanoscratching process are identified by the common neighbor analysis (CNA). ${ }^{29}$ The coloring scheme is designed as blue stands for FCC atoms, green for hexagonal-close-packed (HCP) atoms, and red for Other atoms including surface atoms, grain boundary atoms, and dislocation cores. Here, a single HCP-coordinated-layer identifies a coherent $\mathrm{TB}$, two adjacent $\mathrm{HCP}$-coordinated-layers indicate an intrinsic stacking fault (ISF), two HCP-coordinated-layers with a FCC-coordinated-layer between them represent an extrinsic stacking fault (ESF). ${ }^{30}$ The Atomeye is utilized to visualize MD data and generate MD snapshots. ${ }^{31}$

\section{RESULTS AND DISCUSSION}

\section{A. Scratching-induced deformation twinning}

In the nanoscratching process, the mechanical force acting on the probe can be divided into three components, as scratching force along $\mathrm{X}$ direction, indentation force along $\mathrm{Y}$ direction, and lateral force along $\mathrm{Z}$ direction, respectively. Prior to nanoscratching, the probe is primarily placed above the centre of the surface of a grain in each nanocrystalline $\mathrm{Cu}$ sample. The white arrow in Fig. 2(b) indicates the grain to be penetrated in Sample 2, and the undeformed GBs are highlighted by the white lines. Figure 2(a) plots the indentation force-indentation depth curve of the indentation on Sample 2, indicating the indentation stage can be divided into the following four phases: phase I - elastic deformation, phase II - dislocation activity governed plastic deformation, phase III - TB migration dominated plastic deformation, and phase IV - plastic deformation under the competition of multiple deformation mechanisms such as dislocation activity, GB-associated mechanism, and deformation twinning. To facilitate the analysis of the deformation behavior of $\mathrm{Cu}$ samples, Fig. 3 presents a statistical description of the evolutions of atoms in different lattice environments during the indentation on Sample 2. The reduced number is calculated by subtracting the base number before indentation using the measured 

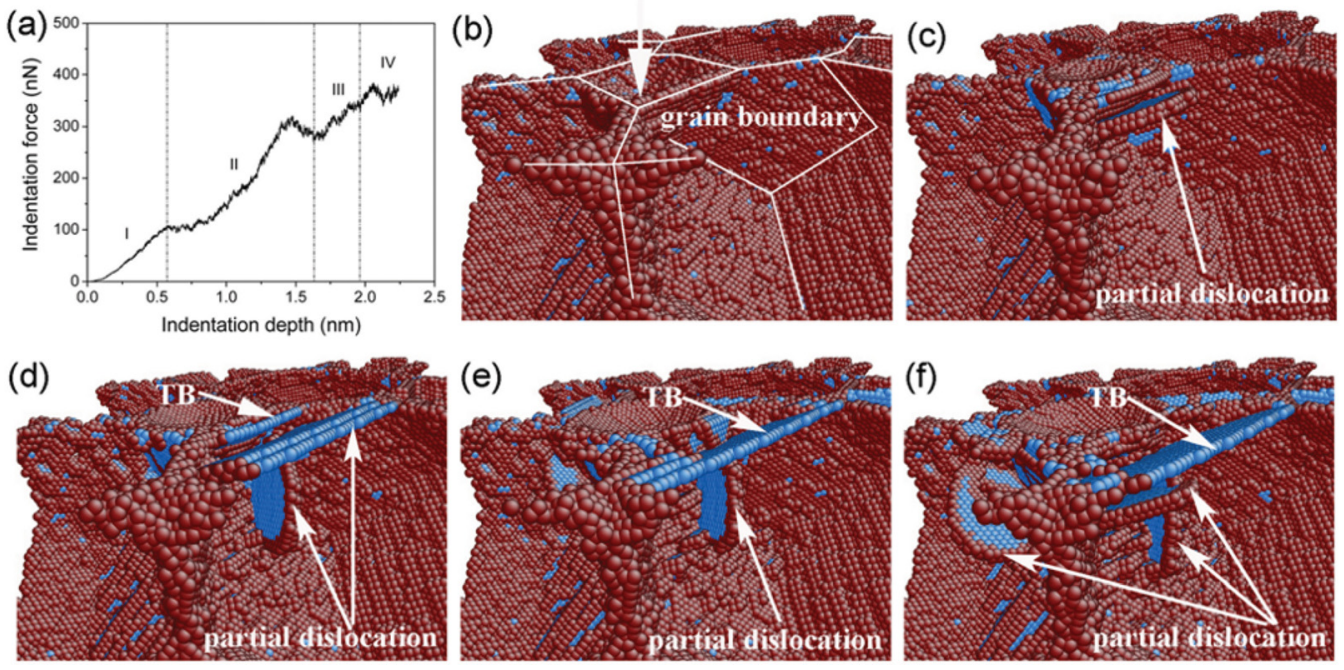

FIG. 2. Indentation on Sample 2. (a) Indentation force versus indentation depth curve. (b)-(f) present representative MD snapshots of instantaneous defect structures obtained at different indentation depths. (b) $0.0 \mathrm{~nm}$, (c) $1.02 \mathrm{~nm}$, (d) $1.42 \mathrm{~nm}$, (e) $1.83 \mathrm{~nm}$, and (f) $2.24 \mathrm{~nm}$. Atoms are colored according to calculated CNA values, and FCC atoms are not shown.

number. The TB atoms are distinguished from ISF atoms by employing the DXA algorithm. ${ }^{32}$ Note that the ESF atoms are treated as TB atoms in DXA algorithm, as the ESF transformed from ISF will eventually widen to nanosized twin lamellae. ${ }^{33}$

It is seen from Fig. 2(a) that the indentation force increases rapidly in phase $\mathrm{I}$, indicating the $\mathrm{Cu}$ sample undergoes elastic deformation. After the indentation depth reaches a critical value of $0.58 \mathrm{~nm}$, a plateau of the force appears due to the avalanche of initial dislocations nucleated from the indented surface. In phase II, the incipient plasticity of the indented $\mathrm{Cu}$ sample is dominantly controlled by the nucleation and subsequent motion of lattice partial dislocations in the indented and neighboring grain interiors. ${ }^{34,35}$ Figure 3 shows that in phase II the FCC atoms decrease rapidly, accompanied with the increase of ISF atoms and Other atoms. The white arrow in Fig. 2(c) highlights the extended leading partial dislocation that emits from the GB triple junction of a neighboring grain. However, the surrounding GBs impede the glides of lattice dislocations in grain interiors as GBs act as strong obstacles to dislocation motion. In addition

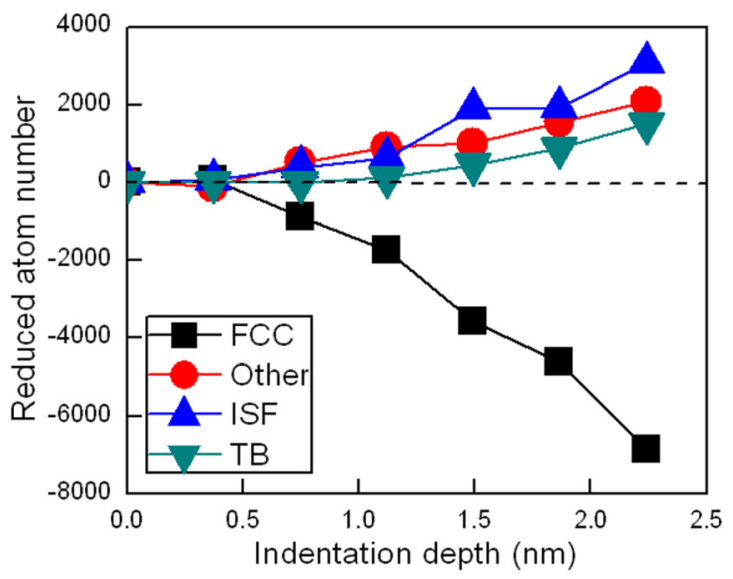

FIG. 3. Evolution of different kinds of atoms during indentation on Sample 2. to the blockage of dislocation motion by GBs, the formation of sessile dislocation structure due to dislocation reaction and cross slip, as shown in Fig. 2(d), also contributes significantly to the work-hardening of the indented material. Consequently, there is high concentrated stress accumulated in the indented region. Upon indentation, one TB, namely $\mathrm{TB} 1$, forms in a neighboring grain via dissociation of the lattice partial dislocation that emits from the GB, accompanied with dramatic decrease of indentation force in phase II. ${ }^{9,36}$ Accordingly, Fig. 3 shows that the TB atoms increase clearly in the later period of phase II. Immediately after nucleation, the twin plane moves downwards an atomic step via TB migration to become stable, as shown in Fig. 2(d). In phase III, another TB, namely TB2, forms in another neighboring grain via the same twinning mechanism.

Figure 3 shows that in phase III, the number of ISF atoms is nearly unchanged, accompanied with a significant increase in the number of TB atoms and Other atoms, implying that the plastic deformation is mediated by other deformation modes rather than dislocation activity. A dynamic analysis of defect evolutions demonstrates that TB migration plays a dominant role in the plastic deformation in phase III. To demonstrate the migration process of TB1, Fig. 4 presents a set of MD snapshots of instantaneous defect structures obtained at different indentation depths. Figures 4(b), 4(g), and 4(h) jointly reveal that TB migration is associated with the nucleation of lattice partial dislocations from TB-GB intersection and their consequent glide on the twin plane. This finding validates the previous experimental observation. ${ }^{37}$ It is seen from Fig. 4(c) that after the TB migrates to reach the partial dislocation with its slip plane parallel to the twin plane, de-twinning occurs and a three-HCP-coordinated-layer is then formed. The three-HCP-coordinated-layer serves as a twin embryo, ${ }^{38}$ because it dissociates into twinning dislocations immediately after its formation, as shown in Figs. 4(d) and 4(e). The plastic deformation of the indented material in phase IV is governed by dislocation activity, GB-associated deformation, de-twinning, and TB 

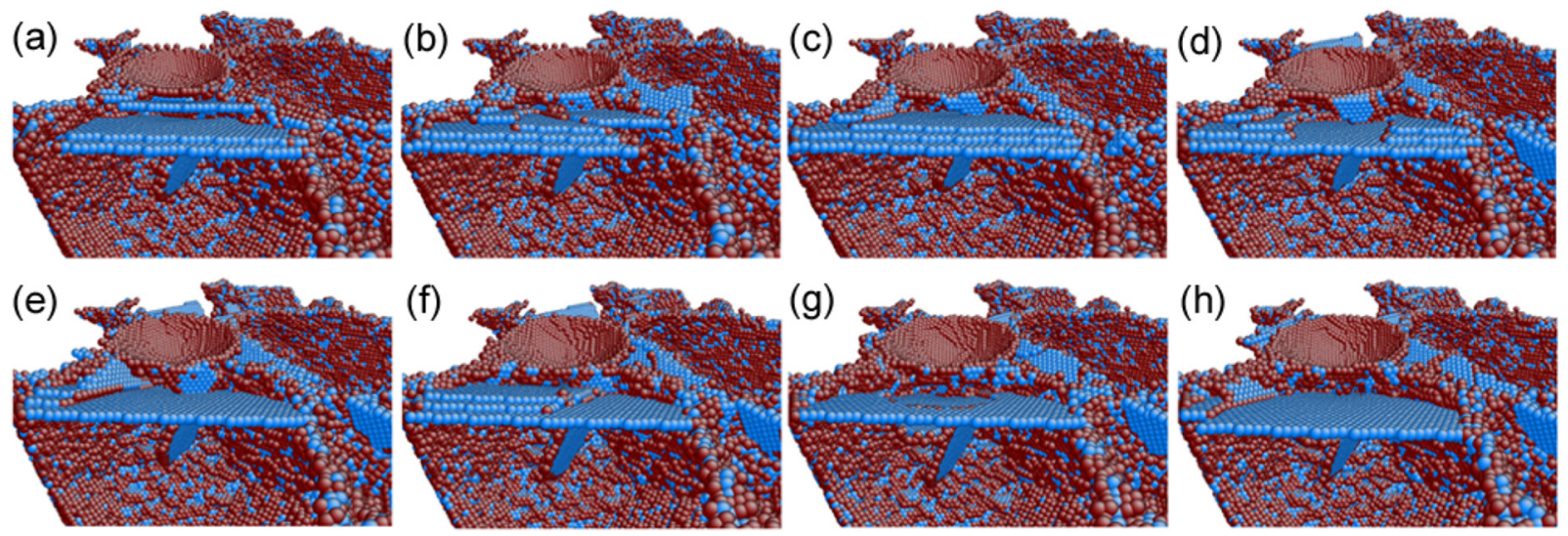

FIG. 4. TB migration during indentation on Sample 2. (a)-(h) present a set of MD snapshots of instantaneous defect structures obtained at different indentation depths. (a) $1.51 \mathrm{~nm}$, (b) $1.59 \mathrm{~nm}$, (c) $1.71 \mathrm{~nm}$, (d) $1.81 \mathrm{~nm}$, (e) $1.85 \mathrm{~nm}$, (f) $2.06 \mathrm{~nm}$, (g) $2.15 \mathrm{~nm}$, and (h) $2.24 \mathrm{~nm}$. Atoms are colored according to calculated CNA values, and FCC atoms are eliminated.

migration working in parallel. It is seen from Fig. 3 that the slope of ISF atom increase in phase IV is larger than both $\mathrm{TB}$ atoms and Other atoms, indicating that the change in GBs, i.e., GBs breaking and grain coalescence, is pronounced. Figure 4(f) shows that de-twinning indeed results from the subsequent glide of lattice partial dislocation on the twin plane after emitting from the TB-GB intersection. In contrast, the following reformation of TBs occurs accompanied by the adsorption of lattice partial dislocations by GBs. Figures 4(g) and 4(h) show a TB undergoing and after migration, respectively. The completed processes of TB migration and de-twinning can be found in Supplementary Video $1 .^{42}$ Figure 5(a) presents the atomic stacking sequence of the
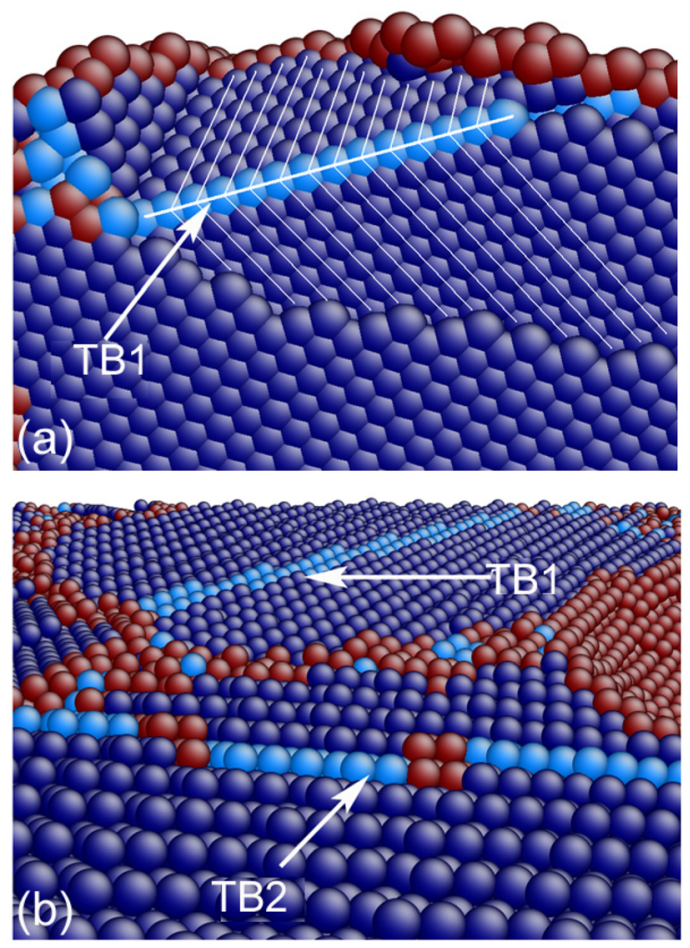

FIG. 5. Deformation twins formed during indentation on Sample 2. (a) The mirror symmetry of stacking sequence across the TB1; (b) uncompleted migration of TB2. Atoms are colored according to calculated CNA values. twin plane shown in Fig. 4(h). In addition, Fig. 5(b) shows the un-completed migration of TB2, which is separated into three ledges connected by twinning partials, in another neighboring grain.

With the fundamental understanding of the indentationinduced deformation of nanocrystalline $\mathrm{Cu}$ obtained in above section, the deformation mechanisms of the $\mathrm{Cu}$ samples in following scratching stage are analyzed in a similar manner. Figure 6(a) plots the scratching force-scratching length curve of the scratching on Sample 2, indicating there are three phases existing in the scratching stage: phase I - dislocation activity governed plastic deformation, phase II - TB migration dominated plastic deformation, and phase III - plastic deformation under the competition between dislocation activity and GB-associated mechanism. We note that the material undergoes more complex deformation in the scratching stage than the indentation stage due to the multi-axial stress states, which induces difficulties to distinguish fractional contributions of individual deformation mechanisms to the macroscopically observed friction response. The statistical description of the evolutions of atoms in different lattice environments during the scratching on Sample 2 is presented in Fig. 7. The reduced number is calculated by subtracting the base number before scratching using the measured number.

In phase I, new lattice partial dislocations nucleate from both the surface and GBs in front of the probe and subsequently glide in grain interiors, as shown in Fig. 6(d). Simultaneously, the release of the compression stress behind the probe leads to annihilation of dislocations generated in former indentation stage and recovery of the indented surface. ${ }^{26}$ Figure 7 shows that the increase of FCC atoms is accompanied with the decrease in both ISF atoms and Other atoms in phase I, indicating that the dislocation annihilation is more pronounced than the nucleation of dislocation. Furthermore, the third TB, namely TB3, forms in a neighboring grain and resides behind the probe, as shown in Fig. 6(e). Accordingly, Fig. 7 shows that the number of TB atoms increases in phase I. Dynamics analysis of defect evolution demonstrates that the plastic deformation in phase II is dominated by the reversible migration of the TB3, which is caused by the 

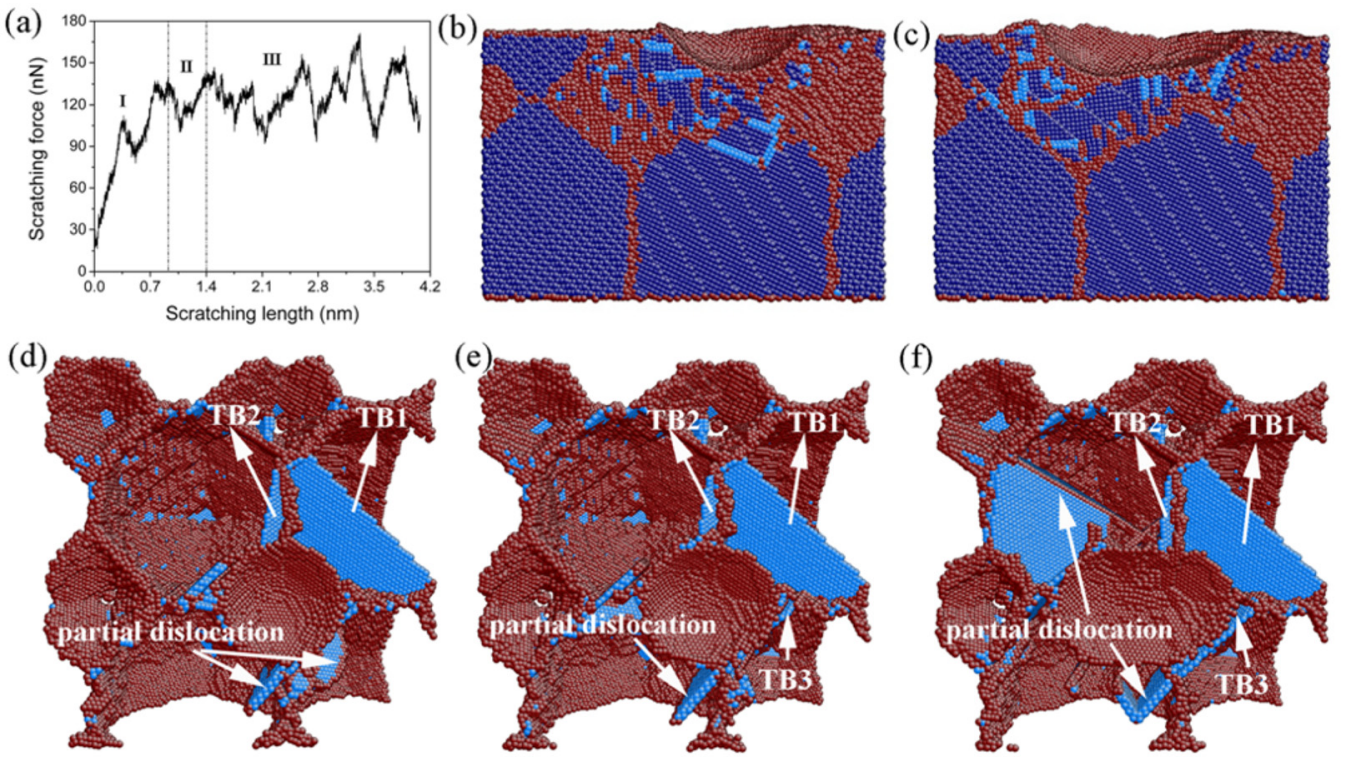

FIG. 6. Scratching on Sample 2. (a) Scratching force versus scratching length curve. (b) and (c) MD snapshots of cross-sectional views of the sample at different scratching lengths. (b) $0.0 \mathrm{~nm}$ and (c) $4.07 \mathrm{~nm}$. (d)-(f) present MD snapshots of top views of instantaneous defect structures at different scratching lengths. (d) $0.41 \mathrm{~nm}$, (e) $1.22 \mathrm{~nm}$, and (f) $2.66 \mathrm{~nm}$. Atoms are colored according to calculated CNA values.

reversible glide of lattice partial dislocations that successively nucleate from both of the two opposite TB-GB intersections on the twin plane. In phase III, there exists a strong competition between dislocation activity and GB-associated mechanism, while the previously formed three TBs keep stable. Upon further scratching, new dislocations successively emit in front of the probe from GBs and then glide in grain interiors and correspondingly in the initial period of phase III, the number of ISF atoms increases accompanied with decrease of FCC atoms, as shown in Fig. 7. However, the adsorption of dislocations by GBs leads to the increase of FCC atoms. With the advancement of the scratching length, more dislocations are nucleated to sustain the deformation induced by the probe, and their reactions and cross slip lead to the formation of the stair-rod dislocation in grain interior shown in Fig. 6(f). Simultaneously, the GBs also undergo significant change. A comparison between Figs. 6(b) and 6(c) reveals that while dislocation annihilation behind the probe leads to the recovery of broken GBs, there are sub-

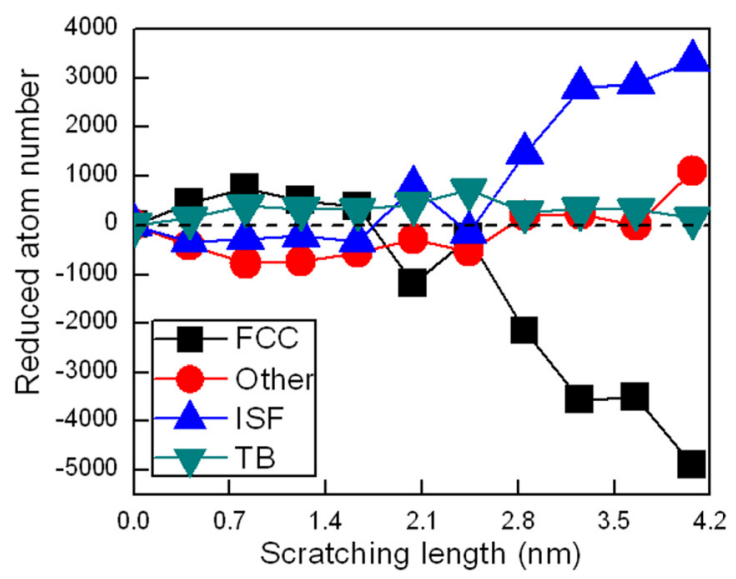

FIG. 7. Evolution of different kinds of atoms during scratching on Sample 2. grains formed in front of the probe to accommodate severe plastic deformation of the material by increasing active slip systems.

\section{B. Grain size effect}

To characterize the grain size dependence of indentation response of nanocrystalline $\mathrm{Cu}$ samples, the indentation hardness defined as the ratio of indentation force to contact area is also calculated. The detailed description about the calculation of contact area during spherical indentation simulation can be found elsewhere. ${ }^{39}$ Figure 8 (a) plots indentation hardness versus indentation depth curves of indentation on nanocrystalline $\mathrm{Cu}$ samples with different grain sizes. It is seen from Fig. 8(a) that the three curves exhibit similar features: the indentation hardness first increases rapidly to reach the maximum value in the elastic regime, and then drops dramatically at the elastic-plastic transmission point due to the initiation of plasticity accompanied by dislocation nucleation. However, the maximum strength of the $\mathrm{Cu}$ samples strongly depends on grain size, and it decreases with decreasing grain size in a reverse Hall-Petch manner. Figure 8(a) also shows that grain size has little influence on the stable value of indentation hardness that is used to compare with experimental data. This may be attributed to the strong competition between individual deformation mechanisms in the final period of indentation stage.

In addition to indentation response, grain size also has strong influence on the following scratching response of $\mathrm{Cu}$ samples. Figure 8(b) plots the friction coefficient versus scratching length curves of scratching on the nanocrystalline $\mathrm{Cu}$ samples with different grain sizes. The friction coefficient follows the normal definition as the ratio of scratching force to indentation force. It is seen from Fig. 8(b) that for each $\mathrm{Cu}$ sample, the friction coefficient first increases rapidly in the initial period of scratching stage, and then fluctuates around 

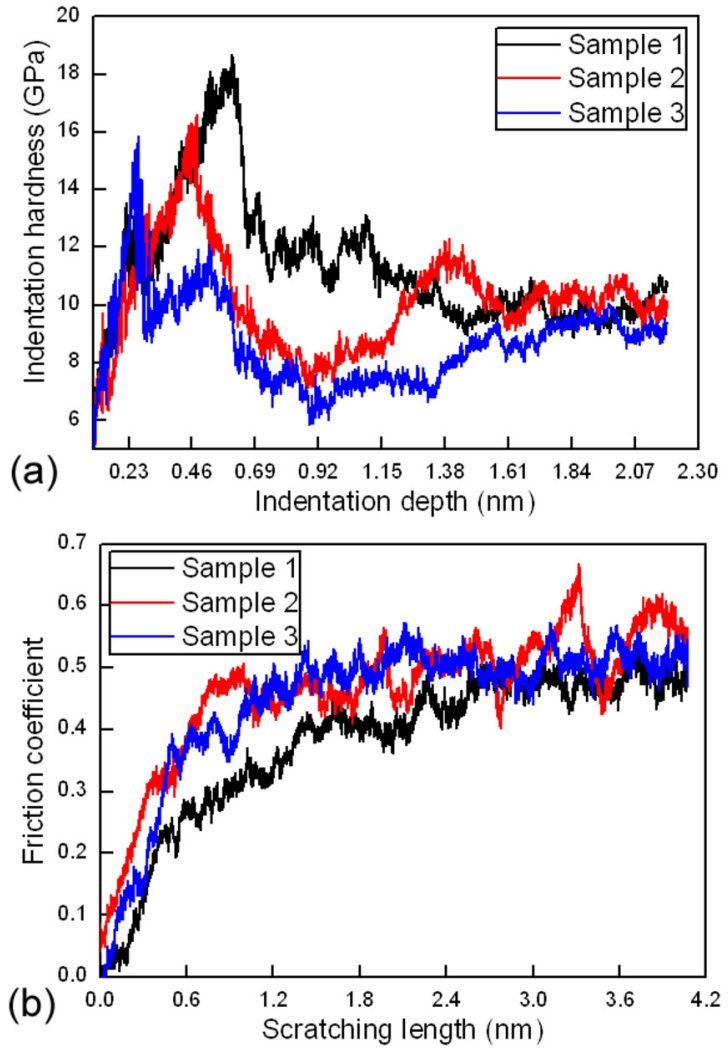

FIG. 8. Effect of grain size on mechanical responses of nanocrystalline $\mathrm{Cu}$ samples during nanoscratching. (a) indentation hardness versus indentation depth curves. (b) Friction coefficient versus scratching length curves.

a constant value when scratching is stable. However, the friction coefficient is smaller for Sample 1 than the other two samples when scratching length is less than $2.65 \mathrm{~nm}$. This discrepancy can be interpreted by the GB proximity effect. Scratching is mainly performed in grain interior of Sample 1 of large grain size, which requires less energy for material removal than scratching on GBs due to dislocation-GB interaction. ${ }^{40}$ Upon further scratching, however, the friction coefficient of Sample 1 increases when the probe is approaching the GB and reaches the same value with other two samples when the probe scratches across the GB. It is indicated that grain size has a significant influence on involved deformation mechanisms, which subsequently affects the mechanical responses of nanocrystalline $\mathrm{Cu}$ under nanoscratching.

To characterize the grain size dependence of the propensity of deformation twinning, the number of TBs formed in indentation and following scratching stages on nanocrystalline $\mathrm{Cu}$ samples with different grain sizes is countered. Note that the TB ledges connected by twinning partials formed due to un-completed TB migration, as shown in Fig. 5(b), are treated as one TB. Furthermore, the ESFs are not taken into account. Figure 9(a) presents the three TBs, highlighted by white arrows, formed after scratching on Sample 2. Figure 9(a) also demonstrates that the plastic deformation behavior varies significantly upon grain orientation. While the plastic deformation in the grain containing TB2 is dominated by deformation twinning, nucleation of lattice partials from GBs is the major deformation mode in the grain containing TB3. This heterogeneous deformation behavior of
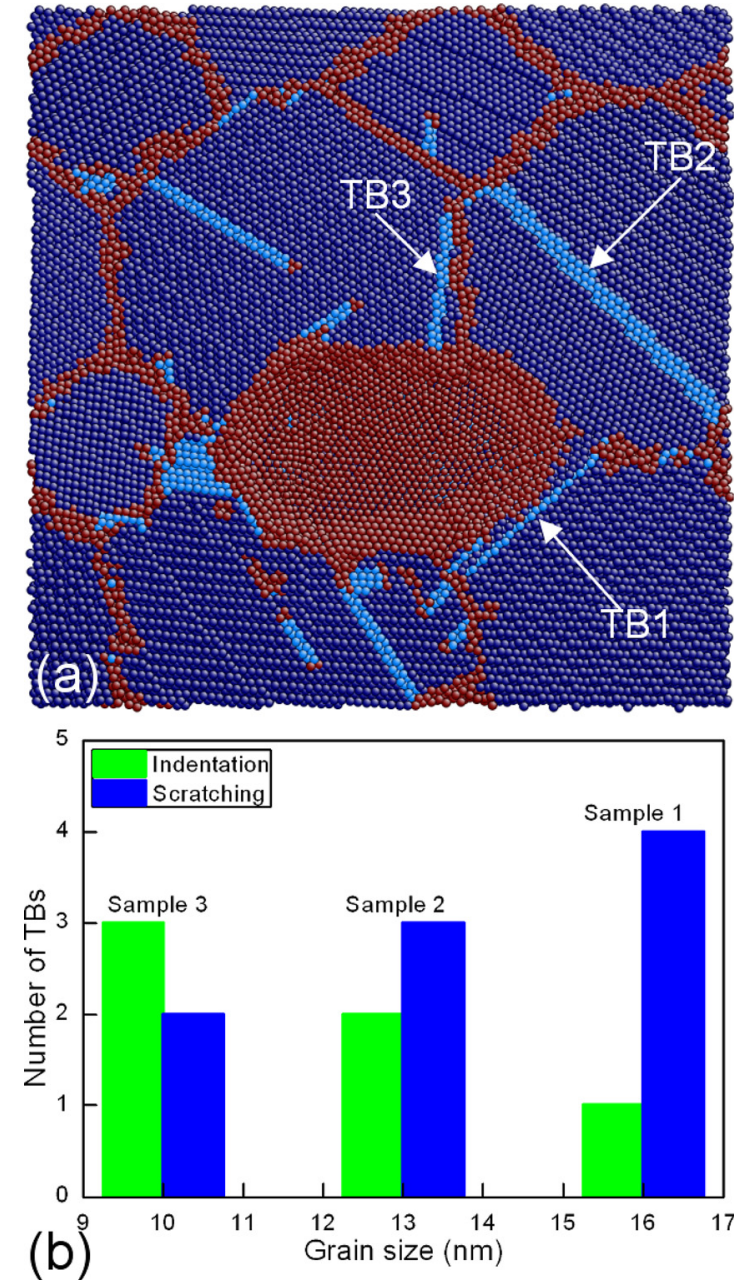

FIG. 9. Grain size dependence of deformation twinning in nanocrystalline $\mathrm{Cu}$ under nanoscratching. (a) MD snapshot of top view of Sample 2 after scratching. Three TBs are identified as highlighted by white arrows. (b) Number of TB formed after indentation and scratching as a function of grain size.

randomly oriented nanocrystalline FCC metals has been reported by previous atomistic simulations. ${ }^{32,41}$ Figure 9(b) plots the number of TBs formed after the completions of indentation and scratching stages as a function of grain size, suggesting that grain size has a strong influence on the propensity of deformation twinning in nanocrystalline $\mathrm{Cu}$ under nanoscratching. After the completion of indentation stage there are 1, 2, and $3 \mathrm{TBs}$ formed in Sample 1, 2, and 3, respectively, indicating a Hall-Petch grain size dependence of deformation twinning. For Sample 1 and 2, Fig. 9(b) shows there are more TBs formed after scratching than indentation. In contrast, the number of TBs in Sample 3 decreases after scratching. It is seen from Fig. 9(b) that there exists a reverse Hall-Petch grain size dependence of deformation twinning in the scratching stage. Note that the as-observed grain size dependence of the propensity of deformation twinning under low temperature of $30 \mathrm{~K}$ may change upon the increase of deformation temperature. ${ }^{12}$

\section{CONCLUSION}

In summary, we perform molecular dynamics simulations to study the deformation mechanisms of nanocrystalline $\mathrm{Cu}$ 
under nanoscratching. Simulation results suggest that in addition to dislocation activity and GB-associated mechanism, deformation twinning, i.e., formation of twin plane, TB migration, and de-twinning, plays an important role in the plastic deformation of nanocrystalline $\mathrm{Cu}$. TBs are formed by the dissociation of lattice partial dislocations that emit from GB triple junctions, and the consequent TB migration is triggered by the emission of lattice partial dislocations from TBGB intersections and their consequent glide on the twin plane. It is found that both grain size and stress state have significant influence on the propensity of deformation twinning in nanocrystalline $\mathrm{Cu}$ under scratching. A Hall-Petch grain size dependence of deformation twinning is observed in indentation stage, while the maximum strength of nanocrystalline $\mathrm{Cu}$ samples decreases with decreasing grain size in a reverse Hall-Petch manner. In the following scratching stage, a reverse Hall-Petch grain size dependence of deformation twinning takes place. The observed remarkable nanoscratching-induced deformation twinning in nanocrystalline $\mathrm{Cu}$ has theoretical and practical significance for the tribology, design, and manufacturing of nanocrystalline $\mathrm{Cu}$ micro/nanosystems.

\section{ACKNOWLEDGMENTS}

J.Z., T.S., Y.Y., and S.D. gratefully acknowledge financial support of China Postdoctoral Science Foundation (2012M511463) and Heilongjiang Postdoctoral Foundation of China (LBH-Z11143), and a Foundation for the Author of National Excellent Doctoral Dissertation of PR China (201031). J.Z. also thanks fruitful discussion with Dr. Alexander Hartmaier. X.L. thanks the support from the University of South Carolina NanoCenter.

${ }^{1}$ E. M. Bringa, A. Caro, Y. M. Wang, M. Victoria, J. M. McNaney, B. A. Remington, R. F. Smith, B. R. Torralva, and H. V. Swygenhoven, Science 309, 1838 (2005).

${ }^{2}$ M. A. Meyers, A. Mishra, and D. J. Benson, Prog. Mater. Sci. 51, 427 (2006).

${ }^{3}$ J. W. Christian and S. Mahajan, Prog. Mater. Sci. 39, 1 (1995).

${ }^{4}$ M. W. Chen, E. Ma, K. J. Hemker, H. W. Sheng, Y. M. Wang, and X. M. Cheng, Science 300, 1275 (2003).

${ }^{5}$ X. Z. Liao, F. Zhou, E. J. Lavernia, D. W. He, and Y. T. Zhu, Appl. Phys. Lett. 83, 5062 (2003).

${ }^{6}$ Y. T. Zhu, X. Z. Liao, S. G. Srinivasan, Y. H. Zhao, M. I. Baskes, F. Zhou, and E. J. Lavernia, Appl. Phys. Lett. 85, 5049 (2004).

${ }^{7}$ A. G. Froseth, P. M. Derlet, and V. H. Swygenhoven, Adv. Eng. Mater. 7, 16 (2005).

${ }^{8}$ T. Zhu, J. Li, A. Samanta, H. G. Kim, and S. Suresh, Proc. Natl. Acad. Sci. U.S.A. 104, 3031 (2007).
${ }^{9}$ M. J. Buehler, Atomistic Modeling of Materials Failure (Springer, New York, 2008) p. 411.

${ }^{10}$ L. Liu, J. Wang, S. K. Gong, and S. X. Mao, Phys. Rev. Lett. 106, 175504 (2011).

${ }^{11}$ P. Gu, M. Dao, R. J. Asaro, and S. Suresh, Acta Mater. 59, 6861 (2011).

${ }^{12}$ Y. T. Zhu, X. Z. Liao, and X. L. Wu, Prog. Mater. Sci. 57, 1 (2012).

${ }^{13}$ X. Z. Liao, Y. H. Zhao, S. G. Srinivasan, Y. T. Zhu, R. Z. Valiev, and D. V. Gunderov, Appl. Phys. Lett. 84, 592 (2004).

${ }^{14}$ Y. T. Zhu, X. Z. Liao, and R. Z. Valiev, Appl. Phys. Lett. 86, 103112 (2005).

${ }^{15}$ C. X. Huang, K. Wang, S. D. Wu, Z. F. Zhang, G. Y. Li, and S. Li, Acta Mater. 54, 655 (2006).

${ }^{16}$ A. J. Cao and Y. G. Wei, Appl. Phys. Lett. 89, 041919 (2006).

${ }^{17}$ X. Y. Li, Y. J. Wei, L. Lu, K. Lu, and H. J. Gao, Nature 464, 877 (2010).

${ }^{18}$ M. A. Meyers, O. Vöhringer, and V. A. Lubarda, Acta Mater. 49, 4025-39 (2001).

${ }^{19}$ N. C. Broedling, A. Hartmaier, M. J. Buehler, and H. J. Gao, J. Mech. Phys. Solids 56, 1086 (2008).

${ }^{20}$ L. Lu, X. Chen, X. Huang, and K. Lu, Science 323, 607 (2009).

${ }^{21}$ E. EI-Danaf, S. R. Kalidindi, and R. D. Doherty, Metall. Mater. Trans. A 30, 1223 (1999).

${ }^{22}$ X. L. Wu and Y. T. Zhu, Phys. Rev. Lett. 101, 025503 (2008).

${ }^{23}$ J. Y. Zhang, G. Liu, R. H. Wang, J. Li, J. Sun, and E. Ma, Phys. Rev. B 81, 172104 (2010).

${ }^{24}$ Y. Mishin, M. J. Mehl, D. A. Papaconstantopoulos, A. F. Voter, and J. D. Kress, Phys. Rev. B 63, 224106 (2001).

${ }^{25}$ E. Bitzek, P. Koskinen, F. Gähler, and M. Moseler, Phys. Rev. Lett. 97, 170201 (2006).

${ }^{26}$ J. J. Zhang, T. Sun, A. Hartmaier, and Y. D. Yan, Comput. Mater. Sci. 59, 14 (2012).

${ }^{27}$ C. L. Kelchner, S. J. Plimpton, and J. C. Hamilton, Phys. Rev. B 58, 11085 (1998).

${ }^{28}$ J. Stadler, R. Mikulla, and H. R. Trebin, Int. J. Mod. Phys. C 8, 1131 (1997); See http://imd.itap.physik.uni-stuttgart.de/ for more details about the IMD code.

${ }^{29}$ J. D. Honeycutt and H. C. Andersen, J. Phys. Chem. 91, 4950 (1987).

${ }^{30}$ V. Yamokov, D. Wolf, S. R. Phillpot, and H. Gleiter, Acta Mater. 50, 5005 (2002).

${ }^{31}$ J. Li, Modell. Simul. Mater. Sci. Eng. 11, 173 (2003).

${ }^{32}$ A. Stukowski and K. Albe, Modell. Simul. Mater. Sci. Eng. 18, 085001 (2010).

${ }^{33}$ A. Stukowski, K. Albe, and D. Farkas, Phys. Rev. B 82, 224103 (2010).

${ }^{34}$ X. L. Ma and W. Yang, Nanotechnology 14, 1208 (2003).

${ }^{35}$ C. Begau, A. Hartmaier, E. P. George, and G. M. Pharr, Acta Mater. 59, 934 (2011).

${ }^{36}$ J. Wang and H. C. Huang, Appl. Phys. Lett. 85, 5983 (2004).

${ }^{37}$ Y. B. Wang, M. L. Sui, and E. Ma, Philos. Mag. Lett. 87, 935 (2007).

${ }^{38}$ S. Mahajan and G. Y. Chin, Acta Metall. 21, 1353 (1973).

${ }^{39}$ G. Ziegenhain, H. M. Urbassek, and A. Hartmaier, J. Appl. Phys. 107, 061807 (2010).

${ }^{40}$ E. T. Lilleodden, J. A. Zimmerman, S. M. Foiles, and W. D. Nix, J. Mech. Phys. Solids. 51, 901 (2003).

${ }^{41}$ X. Y. Li, Y. J. Wei, W. Yang, and H. J. Gao, Proc. Natl. Acad. Sci. U.S.A. 106, 16108 (2009).

${ }^{42}$ See supplementary material at http://dx.doi.org/10.1063/1.4757937 for dynamic process of twin boundary migration and de-twinning. 